\title{
Macrophagic and microglial responses after focal traumatic brain injury in the female rat
}

\author{
L Christine Turtzo ${ }^{1,2^{*}}$, Jacob Lescher ${ }^{1,2}$, Lindsay Janes ${ }^{1,2}$, Dana D Dean ${ }^{2,4}$, Matthew D Budde ${ }^{2,5}$ \\ and Joseph A Frank ${ }^{1,2,3}$
}

\begin{abstract}
Background: After central nervous system injury, inflammatory macrophages (M1) predominate over anti-inflammatory macrophages (M2). The temporal profile of M1/M2 phenotypes in macrophages and microglia after traumatic brain injury (TBI) in rats is unknown. We subjected female rats to severe controlled cortical impact (CCl) and examined the postinjury M1/M2 time course in their brains.
\end{abstract}

Methods: The motor cortex ( $2.5 \mathrm{~mm}$ left laterally and $1.0 \mathrm{~mm}$ anteriorly from the bregma) of anesthetized female Wistar rats (ages 8 to 10 weeks; $N=72$ ) underwent histologically moderate to severe $C C l$ with a 5 -mm impactor tip. Separate cohorts of rats had their brains dissociated into cells for flow cytometry, perfusion-fixed for immunohistochemistry $(\mathrm{IHC})$ and ex vivo magnetic resonance imaging or flash-frozen for RNA and protein analysis. For each analytical method used, separate postinjury times were included for 24 hours; 3 or 5 days; or 1, 2, 4 or 8 weeks.

Results: By IHC, we found that the macrophagic and microglial responses peaked at 5 to 7 days post-TBI with characteristics of mixed populations of M1 and M2 phenotypes. Upon flow cytometry examination of immunological cells isolated from brain tissue, we observed that peak M2-associated staining occurred at 5 days post-TBI. Chemokine analysis by multiplex assay showed statistically significant increases in macrophage inflammatory protein $1 a$ and keratinocyte chemoattractant/growth-related oncogene on the ipsilateral side within the first 24 hours after injury relative to controls and to the contralateral side. Quantitative RT-PCR analysis demonstrated expression of both M1- and M2-associated markers, which peaked at 5 days post-TBI.

Conclusions: The responses of macrophagic and microglial cells to histologically severe CCI in the female rat are maximal between days 3 and 7 postinjury. The response to injury is a mixture of M1 and M2 phenotypes.

Keywords: Controlled cortical impact, Inflammation, Macrophage, Microglia, MRI, Rat, Traumatic brain injury

\section{Background}

Traumatic brain injury (TBI) is responsible for $50 \%$ of trauma-related deaths in the United States and accounts for a substantial burden of disability in survivors [1,2]. To date, few effective treatments for acute TBI or its long-term sequelae have been identified [3]. The initial trauma to the brain triggers an inflammatory response,

\footnotetext{
*Correspondence: turtzolc@mail.nih.gov

${ }^{1}$ Center for Neuroscience and Regenerative Medicine, Uniformed Services University of the Health Sciences, 4301 Jones Bridge Road, Bethesda, MD 20814, USA

${ }^{2}$ Frank Laboratory, National Institutes of Health, Building 10, Room B1N256, MSC 1074, 10 Center Drive, Bethesda, MD 20814, USA

Full list of author information is available at the end of the article
}

in which both resident microglia and peripheral macrophages play key roles [4].

Similar to the phenotypes of $\mathrm{T}$ cells in autoimmune and inflammatory diseases, different subsets of peripheral macrophages (inflammatory macrophages (M1) and antiinflammatory macrophages (M2a and M2c)) have been identified in gliomas [5] and in breast and lung cancer $[6,7]$, as well as after injury caused by acute peripheral nerve transection [8] and spinal cord injury [9]. The proinflammatory M1 phenotype is associated with tissue destruction $[6,7,9,10]$, whereas the anti-inflammatory M2a and M2c phenotypes facilitate repair and regeneration in part by reducing inflammatory mediators $[6,9,11]$. 
Macrophages and microglia do not exist as immutable subsets; rather, they are sensitive to their host tissue microenvironments, with their phenotypes determined in part by their surroundings and the length of time after injury [9,12-15]. Macrophages can be differentiated in vitro into an M1 phenotype by lipopolysaccharide and interferon $\gamma($ IFN- $\gamma)$ [16], resulting in classically activated macrophages that generate proinflammatory cytokines (for example, tumor necrosis factor $\alpha$ (TNF $\alpha)$ ). The M2 subset results from activation of macrophages with interleukin 4 (IL-4) or IL-13 and can support reparative and regenerative processes $[10,11,17,18]$.

The temporal relationship driving inflammatory and/or neurodegenerative processes versus reparative and/or neuroregenerative events following acute neurotrauma may be influenced by the ratio of M1 to M2 phenotypes [9-11,19]. Whether a similar schism exists in microglial and macrophagic phenotypes in the response to experimental TBI in the rat is currently unknown. We hypothesized that, after focal cortical contusion in the rat, larger numbers of M1 than M2 macrophages and microglia would be located within and around traumatic lesions.

The purpose of this study was to perform a timecourse analysis of the macrophagic and microglial responses after TBI in the female rat. Tissue samples taken from brains postinjury were analyzed for expression of macrophagic and microglial markers by immunohistochemistry (IHC), flow cytometry and RNA and protein analysis. The results suggest that the postinjury environment results in a mixed population of microglia and macrophages rather than a milieu that is exclusively proor anti-inflammatory.

\section{Methods}

\section{Animals}

All studies were approved by the Institutional Animal Care and Use Committee at our institution. Experiments were performed according to the National Research Council's Guide for the Care and Use of Laboratory Animals. Female Wistar rats (Charles River Laboratories, Wilmington, MA, USA) at an initial age of 8 weeks and weighing approximately $200 \mathrm{~g}(N=72)$ were housed in pairs under 12-hour light-dark cycles in temperaturecontrolled conditions. Rats were given water and standard rodent laboratory chow ad libitum.

\section{Controlled cortical impact model}

After the rats were anesthetized by nosecone administration of $2 \%$ to $3 \%$ isoflurane in $100 \%$ oxygen, the rat controlled cortical impact (CCI) model protocol [20-22] was carried out using an electromagnetic device (Impact One Stereotaxic Impactor (Leica Microsystems, Richmond, IL, USA) [23]. As previously described [24], a 6-mm craniotomy was followed by CCI with a $5-\mathrm{mm}$ flat impactor tip over the left motor cortex $(2.5 \mathrm{~mm}$ left lateral and $1.0 \mathrm{~mm}$ anterior from the bregma; velocity $=5 \mathrm{~m} / \mathrm{s}$, depth $=$ $2.5 \mathrm{~mm}$ and dwell time $=100 \mathrm{~ms}$ ). The same surgeon performed all CCI surgeries for a given cohort of rats.

\section{Cell isolation and flow cytometry}

Control and post-CCI rats $(n=24)$ were killed with isoflurane and pentobarbital, then perfused with ice-cold, heparinized phosphate buffered saline (PBS). After each brain extraction, a 200-mg section centered over the injury site (or its equivalent in controls) was removed and placed into ice-cold Hanks' balanced salt solution without calcium or magnesium. Tissues underwent enzymatic and mechanical dissociation with the MACS Neural Tissue Dissociation Kit (P) (Miltenyi Biotec, Bergisch Gladbach, Germany), followed by cell purification by centrifugation in an OptiPrep gradient medium (AxisShield, Oslo, Norway) to remove myelin and debris and to enrich the tissues for brain-derived macrophages and microglia as described previously [25].

After isolation, cells were fixed with BD Cytofix fixation buffer (BD Biosciences, San Jose, CA, USA) according to the manufacturer's instructions. Cells $(100,000$ per reaction) were stained for CD40 (M1 marker), CD68 (generic macrophage marker) and CD163 (M2 marker) using antibodies against CD40 (eBioscience, San Diego, CA, USA) and against CD68 clone ED1 and CD163 (AbD Serotec, Kidlington, UK). Unstained cells and cells stained with appropriate isotype controls for each of the antibodies were used in control experiments. Stained cells and controls were analyzed on an Accuri C6 flow cytometer (BD Biosciences). A total of 30,000 events were counted in windows gated for the intersection of CD68 staining with CD40 and CD163.

\section{Immunohistochemistry}

Rats $(n=24)$ were killed with isoflurane and pentobarbital and perfused with ice-cold, heparinized PBS followed by $4 \%$ paraformaldehyde. After extraction, perfused brains were placed into a sucrose gradient medium, transferred to optimal cutting temperature media and frozen in liquid nitrogen. Coronal plane cryosections (10 $\mu \mathrm{m}$ thick) were cut, mounted on Superfrost Plus glass slides (Thermo Scientific, Rockford, IL, USA) and stored at $-20^{\circ} \mathrm{C}$. Representative sections were examined by hematoxylin and eosin (H \& E) staining using standard methods.

For IHC analysis, slides were washed with PBS, blocked in Super Block (ScyTek Laboratories, Logan, UT, USA) for 10 minutes at room temperature and incubated in primary antibody to detect the following: activated microglia and ionized calcium-binding adapter molecule 1 (Iba1) (Wako Chemicals, Richmond, VA, USA) at 1:200 dilution; or macrophages and CD68 
(Abcam, Cambridge, MA, USA) at 1:200 dilution and CD86 (BD Biosciences) at 1:10 dilution; and CD163 (Hycult Biotech, Plymouth Meeting, PA, USA) at 1:10 dilution in $1 \times$ PBS, $0.3 \%$ Tween 20 and $1.0 \%$ bovine serum albumin at $4^{\circ} \mathrm{C}$ overnight. After being washed three times in PBS with 0.3\% Tween 20, slices were incubated in secondary antibody (for CD68/CD86/CD163: goat $\mathrm{F}(\mathrm{ab})$ secondary polyclonal antibody to mouse immunoglobulin G in H \& L DyLight 649 (Abcam); for Iba-1: goat anti-rabbit F (ab') Alexa Fluor $633(\mathrm{H}+\mathrm{L})$ (Life Technologies, Carlsbad, CA, USA)) at a dilution of $1: 200$ in $1 \times$ PBS with $0.3 \%$ Tween 20 and $1.0 \%$ bovine serum albumin at room temperature for 1 hour, rinsed three times in PBS with $0.3 \%$ Tween 20, dipped in distilled water and mounted in ProLong Gold Antifade reagent with 4',6-diamidino-2-phenylindole (Invitrogen, Carlsbad, CA, USA). Slides were visualized with an Aperio ScanScope FL fluorescence microscope (Aperio/ Leica Microsystems, Vista, CA, USA).

\section{Magnetic resonance imaging}

After perfusion and paraformaldehyde fixation, a subset of the excised rat brains $(n=8)$ from the IHC cohort were immersed in susceptibility matching fluid (Fomblin PFPE lubricant; Solvay Solexis, Inc, West Deptford, NJ, USA) prior to IHC. Brains were imaged using a 7-T Bruker vertical bore magnet (Bruker BioSpin, Billerica, MA, USA) with a $10-\mathrm{mm}$ inner diameter birdcage coil with a threedimensional multiecho gradient echo sequence: repetition time $(\mathrm{TR})=200 \mathrm{~ms}$, effective echo time $(\mathrm{TE})=17.5 \mathrm{~ms}$ (six echoes, $5 \mathrm{~ms}$ echo spacing), field of view $=3.0 \times 1.5 \times$ $1.25 \mathrm{~cm}^{3}, 512 \times 256 \times 128$ matrix, four averages and $30^{\circ}$ flip angle as previously described [24].

\section{Immunofluorescent image analysis}

For each animal, three brain slices per rat were selected from the center of the lesion and stained for a given antibody. Three regions of interest (ROIs) per tissue slice (20x magnification) were selected on the basis of consistent anatomical locations encompassing the medial, ventral and lateral aspects of the perilesion cortex. For each fluorophore, the same intensity thresholds were applied to quantify total fluorescent area using the Aperio ImageScope Positive Pixel Count Algorithm FL version 1 (Aperio/Leica Microsystems). Fluorescence quantification for each ROI was then averaged, divided by the total area of the region of interest $\left(0.3979 \mathrm{~mm}^{2}\right)$ and multiplied by 100 to calculate the percentage fluorescence. Immunofluorescence data were analyzed using Prism Mac version 6.0c software (GraphPad Software, San Diego, CA, USA) by two-way analysis of variance (ANOVA) with the Bonferroni correction using time and ROI location as variables, and by one-way ANOVA with a post hoc Tukey correction with time used as a variable.

\section{RNA and protein extraction}

After rats $(n=24)$ were killed with isoflurane and pentobarbital, their brains were rapidly extracted and placed on ice. A 400-mg wedge of perilesion and lesion of injured cortex and striatum was removed, flash-frozen in liquid nitrogen and stored at $-80^{\circ} \mathrm{C}$. Prior to further extraction, frozen brain tissue was placed into prechilled Ambion RNAlater-ICE reagent (Ambion/Life Technologies, Austin, TX, USA) and kept at $-20^{\circ} \mathrm{C}$ for a minimum of 16 hours in accordance with the manufacturer's instructions. Tissue was homogenized on ice in PARIS Cell Disruption Buffer (Ambion) supplemented with a protease inhibitor (Complete Protease Inhibitor Cocktail; Santa Cruz Biotechnology, Santa Cruz, CA, USA) with Omni Tip probes (Omni International, Kennesaw, GA, USA). RNA and protein fractions were processed from the same starting tissue samples using the Ambion PARIS kit per the manufacturer's instructions and stored at $-80^{\circ} \mathrm{C}$. RNA concentrations and integrity were determined by analysis on Experion RNA StdSens chips (BioRad Laboratories, Hercules, CA, USA). Protein concentrations were determined by bicinchoninic acid assay (Thermo Scientific).

\section{Quantitative RT-PCR}

RNA was transcribed into cDNA using the SABiosciences $\mathrm{RT}^{2}$ First Strand Kit (QIAGEN, Valencia, CA, USA) according to the manufacturer's instructions. Quantitative RT-PCR (qRT-PCR) was performed using the CFX96 Touch Real-Time PCR Detection System (Bio-Rad Laboratories) with SABiosciences $\mathrm{RT}^{2}$ SYBR Green qPCR Master Mix (QIAGEN). Pooled cDNA samples from each time point were first screened with the SABiosciences Rat Common Cytokines $\mathrm{RT}^{2}$ Profiler PCR Array or with specific primers for M1- and M2associated markers (QIAGEN). The expression of genes of interest that showed significant up- or downregulation on this initial screen was then confirmed by running individual samples in triplicate for each time point on custom arrays using validated SABiosciences PCR primers for those rat genes (QIAGEN). Data were analyzed using SABiosciences $\mathrm{RT}^{2}$ Profiler PCR Array Data Analysis version 3.5 software and the SABiosciences $\mathrm{RT}^{2}$ Profiler PCR Array Data Analysis Template version 4.0 (QIAGEN).

\section{Cytokine and chemokine analysis}

Protein samples at concentrations of $2 \mathrm{mg} / \mathrm{ml}$ were analyzed using the Bio-Plex Pro Rat Cytokine 23-plex Assay on a Bio-Plex 200 System and Bio-Plex Pro II Wash 
Station (Bio-Rad Laboratories) per the manufacturer's instructions.

\section{Statistical analysis of RNA and protein data}

Molecular data were analyzed by one-way ANOVA with a subsequent post hoc Tukey test using Prism Mac 6.0c software. A two-tailed $P$ value $<0.05$ was considered to be significant. Errors are expressed as standard errors of the mean.

\section{Results}

To determine the proinflammatory (M1) versus antiinflammatory (M2) profiles of macrophages and microglia in the brain after CCI, we performed a serial timecourse study (Figure 1). The rat brains were harvested for ex vivo magnetic resonance imaging (MRI), and tissues were processed for flow cytometry, IHC and RNA and protein analysis. In the first 24 hours after injury, ex vivo MRI and corresponding $\mathrm{H}$ \& $\mathrm{E}$-stained images demonstrated edema and tissue damage at the injury site (Figure 2). Over time, the damage evolved. Areas of hemorrhage developed by 1 week, which subsequently cleared over time, resulting in a cavity by 4 weeks postinjury that remained unchanged in size at 2 months after injury (data not shown).

Cells isolated from brain tissues for flow cytometry displayed characteristic macrophagic and microglial markers (Figure 3). Expression of the M2 marker CD163 was significantly different relative to baseline at 3 and 5 days postinjury (DPI) (CD163 expression as percentage of cells counted: control $=0.9 \pm 0.2 \%, 3 \mathrm{DPI}=12 \pm 3 \%, 5 \mathrm{DPI}=$ $20 \pm 2 \% ; F=55.70, P \leq 0.0001$ by one-way ANOVA). There were no significant differences in expression of the general macrophagic marker CD68 or the M1 marker CD40 in comparison to control values over the time course.

Immunohistochemical staining for M1 and M2 markers on histological sections revealed a maximum generic macrophagic response (that is, CD68) at 5 DPI (Figure 4) (ROI: 1 DPI $=0.3 \pm 0.1 \%, 5 \mathrm{DPI}=11 \pm 2 \% ; F=15.11, P<$ $0.0001)$. Iba1 staining, characteristic of microglial activation, peaked at 1 week postinjury (ROI: $1 \mathrm{DPI}=1.8 \pm 0.1 \%$, $7 \mathrm{DPI}=19 \pm 4 \% ; \quad F=6.168, P=0.0027)$. M2-associated
CD163 staining was highest at 5 DPI (ROI (CD163): 1 $\mathrm{DPI}=0.22 \pm 0.09 \%, 5 \mathrm{DPI}=7 \pm 1 \% ; F=5.764, P=0.0043)$. Changes in M1-associated CD86 in comparison to $1 \mathrm{DPI}$ were not significantly different by one-way ANOVA (ROI: $1 \mathrm{DPI}=0.3 \pm 0.3 \%, 5 \mathrm{DPI}=7.0 \pm 0.4 \% ; F=1.120, P=0.4$ ). Although a significant effect of time on staining for M1 and M2 markers was observed (two-way ANOVA for time for all markers: $F>4.5$ ), no statistically significant differences were found among ROI locations by two-way ANOVA. Because of the lack of an effect of ROI location, the data are reported as the mean of all ROI locations for a given time point.

Qualitative double-labeling of brain sections for CD68 and CD86 or for CD68 and CD163 demonstrated different localizations of M1 versus M2 macrophages (Figure 5). The CD86-positive CD68 macrophages were located predominantly in the necrotic area of the lesion, and the CD163-positive CD68 positive macrophages were found in the perilesion area.

RNA expression of markers CD68, CD86, Iba1 and mannose receptor, $\mathrm{C}$ type 1 (Mrc1) was highest relative to control and contralateral expression at 5 DPI (fold changes in CD68: control $=1 \pm 0.2,5 \mathrm{DPI}=72 \pm 27(F=$ 4.488, $P=0.0002)$; fold changes in CD86: control $=1 \pm$ $0.2,5 \mathrm{DPI}=14 \pm 6 \quad(F=3.117, P=0.0036)$; fold changes in Iba1: control $=1 \pm 0.5,5 \mathrm{DPI}=5 \pm 3(F=3.720, P=$ 0.0011 ); fold changes in Mrc1: control $=1 \pm 0.1,5 \mathrm{DPI}=$ $12 \pm 4(F=3.976, P=0.0006))$ (Figure 6). TNFo increased significantly at days 1 and 3 after injury (fold changes in TNFo: control $=1 \pm 0.1,24$ hours postinjury $=3.6 \pm 0.6,3$ $\mathrm{DPI}=3.1 \pm 0.6(F=3.9, P=0.0007))$. No statistically significant differences were found in the expression of arginase 1 (Arg1), CD163 or nitric oxide synthase 2 (Nos2). RNA for chemokine (C-X-C motif) ligand 1 (CXCL1), cytokine IL-11 and IL-1 $\beta$ was maximal at 24 hours postinjury (Figure 7) (fold changes in CXCL1: control $=1 \pm$ 0.3 , 24 hours postinjury $=21 \pm 6(F=11.10, P<0.0001)$; fold changes in IL-11: control $=1 \pm 0.3$, 24 hours postinjury $=8 \pm 4(F=3.221, P=0.0031)$; fold changes in IL-1 $\beta$ : control $=1 \pm 0.2, \quad 24$ hours postinjury $=31 \pm 10 \quad(F=$ 7.600, $P<0.0001)$ ). Proinflammatory cytokine RNA for IL-18 peaked at 5 days (fold changes in IL-18: control =

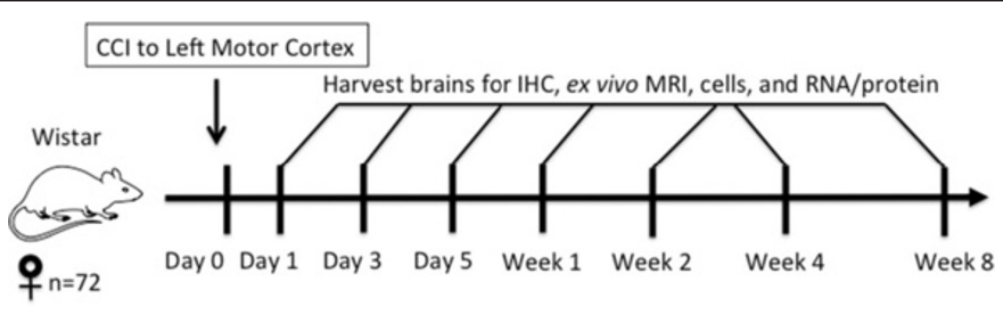

Figure 1 Experimental design. Female Wistar rats $(N=72)$ underwent controlled cortical impact $(\mathrm{CCl})$ to the left motor cortex on day 0 . Their brains were harvested ( $n=9$ per time point) for immunohistochemistry (IHC), ex vivo magnetic resonance imaging (MRI), flow cytometry and RNA/protein analysis over a time course ranging from 1 day to 8 weeks postinjury. Naïve control rats were subjected to the same protocol. 


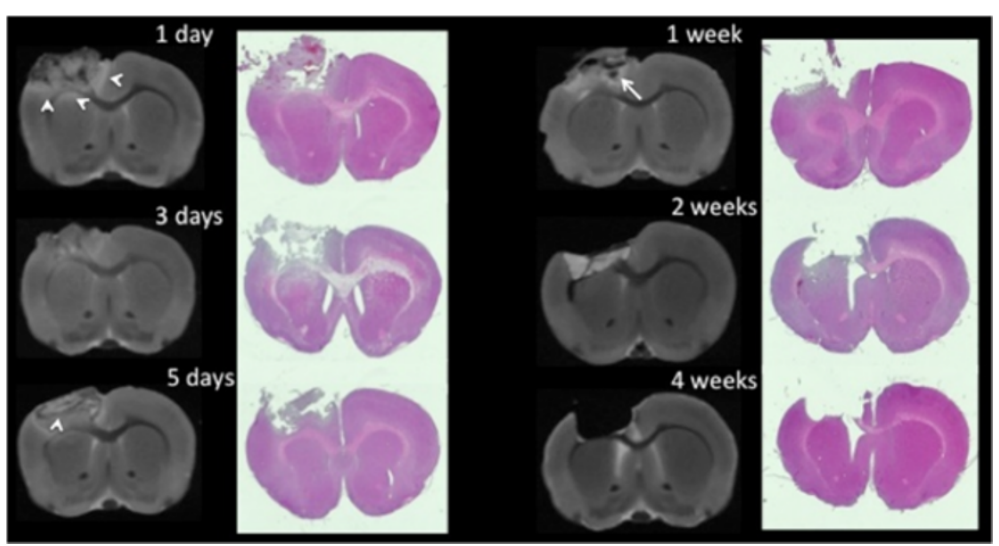

Figure 2 Evolution of controlled cortical impact injury over time. Ex vivo magnetic resonance imaging (MRI) and corresponding hematoxylin and eosin stains demonstrate the controlled cortical impact (CCI) lesion's extension from the cortex through the corpus callosum. By 4 weeks postinjury, the lesion had evolved into a cavity, with minimal further changes observed by 8 weeks (data not shown). Arrowheads on day 1 MRI scans indicate areas of edema. The arrow on the day 7 scan indicates an area of hemorrhage.

$1 \pm 0.2,5 \mathrm{DPI}=4 \pm 2(F=2.688, P=0.0103))$. RNA expression of IL-1 receptor antagonist (IL-1ra), an antiinflammatory cytokine, was elevated 15 -fold from days 1 through 5, with a peak at 3 DPI (fold changes in IL-1ra: control $=1 \pm 0.3,3$ DPI $=23 \pm 5 ; F=7.479, P<0.0001)$.

The multiplex protein profiles of most cytokines from 24 hours through 2 months postinjury showed a statistically significant decrease in ipsilateral side cytokine levels relative to control and in the contralateral side at 24 hours (Figure 8 and Additional file 1: Figure S1). In contrast, macrophage inflammatory protein $1 \alpha$ (MIP$1 \alpha)$ and keratinocyte chemoattractant/growth-related oncogene (KC/GRO, also known as CXCL1) increased significantly relative to control and the contralateral side at 24 hours postinjury $(P<0.05)$.

\section{Discussion}

The following are the major findings of our serial timecourse analysis after CCI in the female rat. (1) The maximum response of microglia and macrophages in the traumatically injured brain occurred within the first week after injury. (2) Markers indicative of pro- and antiinflammatory activity are expressed within the first week after injury. (3) M1 and M2 markers return to nearbaseline levels by 1 month after injury. To the best of our knowledge, our present study is the first in which the M1 and M2 responses of macrophages and microglia after $\mathrm{CCI}$ in female rats have been investigated. Comparisons of findings between published papers are complicated by animal strains used, animal sex, and methodological differences in studies using comparable as well as in different TBI models.

Cole et al. demonstrated that a sham craniotomy itself results in an inflammatory response at $1 \mathrm{DPI}$, with elevation of $\mathrm{KC} / \mathrm{GRO}$ protein levels relative to naïve controls
[26]. Greater changes were seen when the craniotomy was performed by using trephine versus drill. Therefore, for true comparison to baseline levels in the rat in the present study, naïve controls were used instead of craniotomy shams and all craniotomies were performed with drills. Consistent with reports of prior studies in which Sprague-Dawley male rats were used [27], we found activation of microglia in the hemisphere ipsilateral to injury in female Wistar rats after CCI.

By both flow cytometry and IHC, we observed a mixed pattern of M1 and M2 marker expression (Figures 3 and 4). M1 markers were present with the use of both techniques, but we did not find any statistically significant differences over the time course. M2 markers, however, peaked by 5 DPI and then decreased to control levels. Double-labeling experiments indicated that the majority of CD163+ cells localized to the perilesion area, whereas the CD86+ cells were located predominantly within the lesion itself (Figure 5). These observations support the idea that the M1 response is responsible for clearing dead tissue and that the M2 response is to preserve salvageable tissue in the perilesion area.

qRT-PCR analysis demonstrated a mixed pattern of expression for macrophage- and microglia-associated markers. Peak mRNA levels of the M1 markers CD80 and CD86, the M2 marker Mrc1 (CD206), the general macrophagic marker CD68 and the microglial marker Iba1 occurred at $5 \mathrm{DPI}$, with normalization occurring by 2 months after injury (Figure 6). qRT-PCR experiments carried out to study inflammation-related cytokines and chemokines showed a peak TNF $\alpha$ level at 24 hours postinjury, possibly indicative of its upregulation in resident microglia or in other cell types, such as astrocytes [28] or injured neurons [29], as peripheral macrophage infiltration is minimal at that time point. The upregulation 


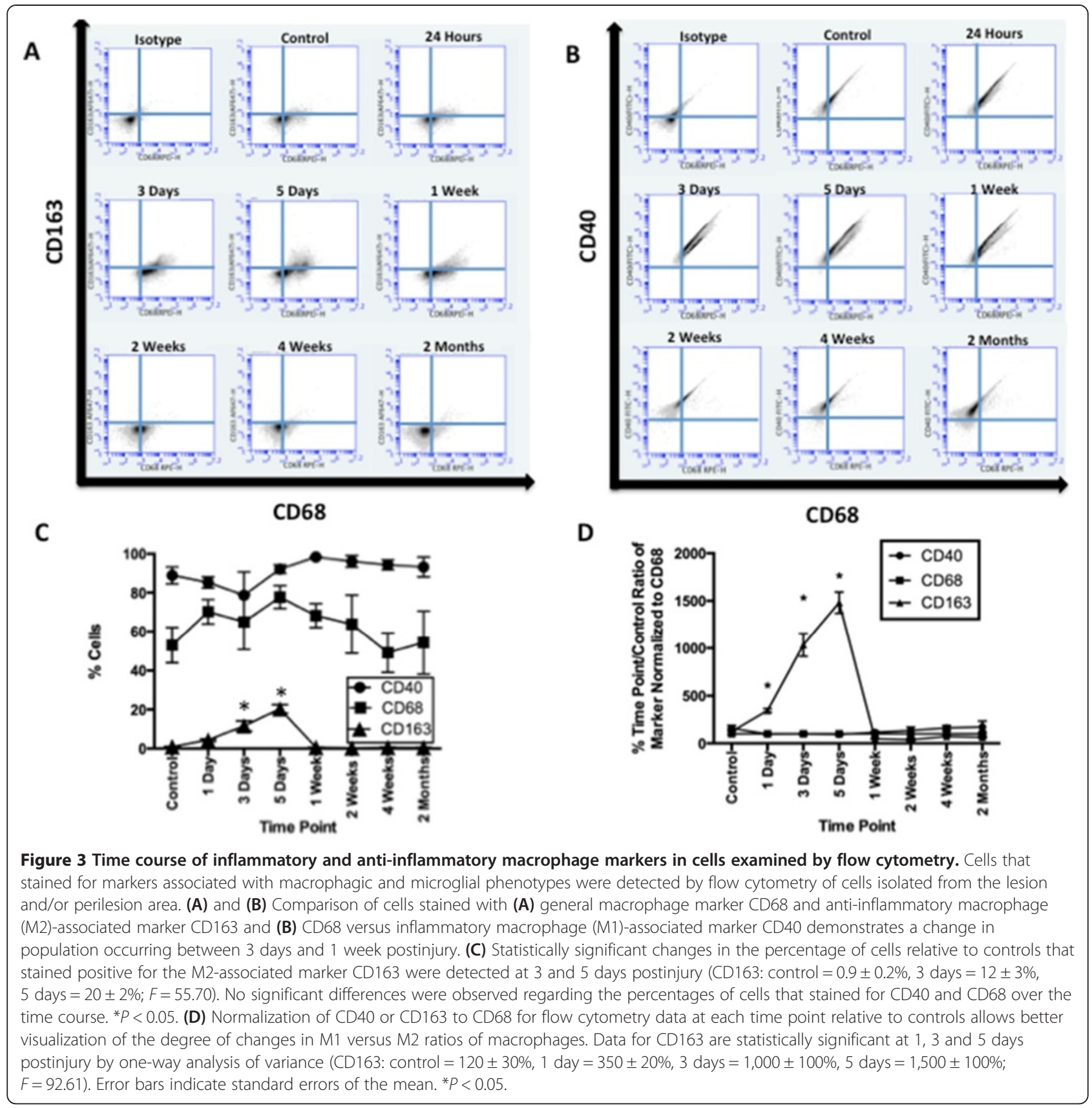

of CXCL1, IL-1 $\beta$ and IL-11 RNA at 24 hours postinjury determined by qRT-PCR (Figure 7) may also be due to the presence of other cell types [30,31].

Analysis of selected cytokines and chemokines by multiplex assays demonstrated an almost universal decrease in cytokine and chemokine levels on the ipsilateral side relative to controls. The exceptions to these data were for proinflammatory chemokines MIP- $1 \alpha$ and $\mathrm{KC} / \mathrm{GRO}$, which showed maximum increases at 24 hours, followed by decreases (Figure 8). The increases in these two chemokines are comparable to those reported by investigators in other studies [32,33].
In the present study, the population of brain macrophages and microglia after TBI shares characteristics of both pro- and anti-inflammatory markers. These results, in combination with those reported by Hsieh et al. [34] and others, suggest that these cells may fluctuate along a continuum between proinflammatory and anti-inflammatory responses, depending upon their environment after injury, rather than being at the extremes of the spectrum. Although some of these "classic" M1 and M2 markers change over the time course, others do not, suggesting that characteristics are more intermediate in these cell types after experimental TBI. 

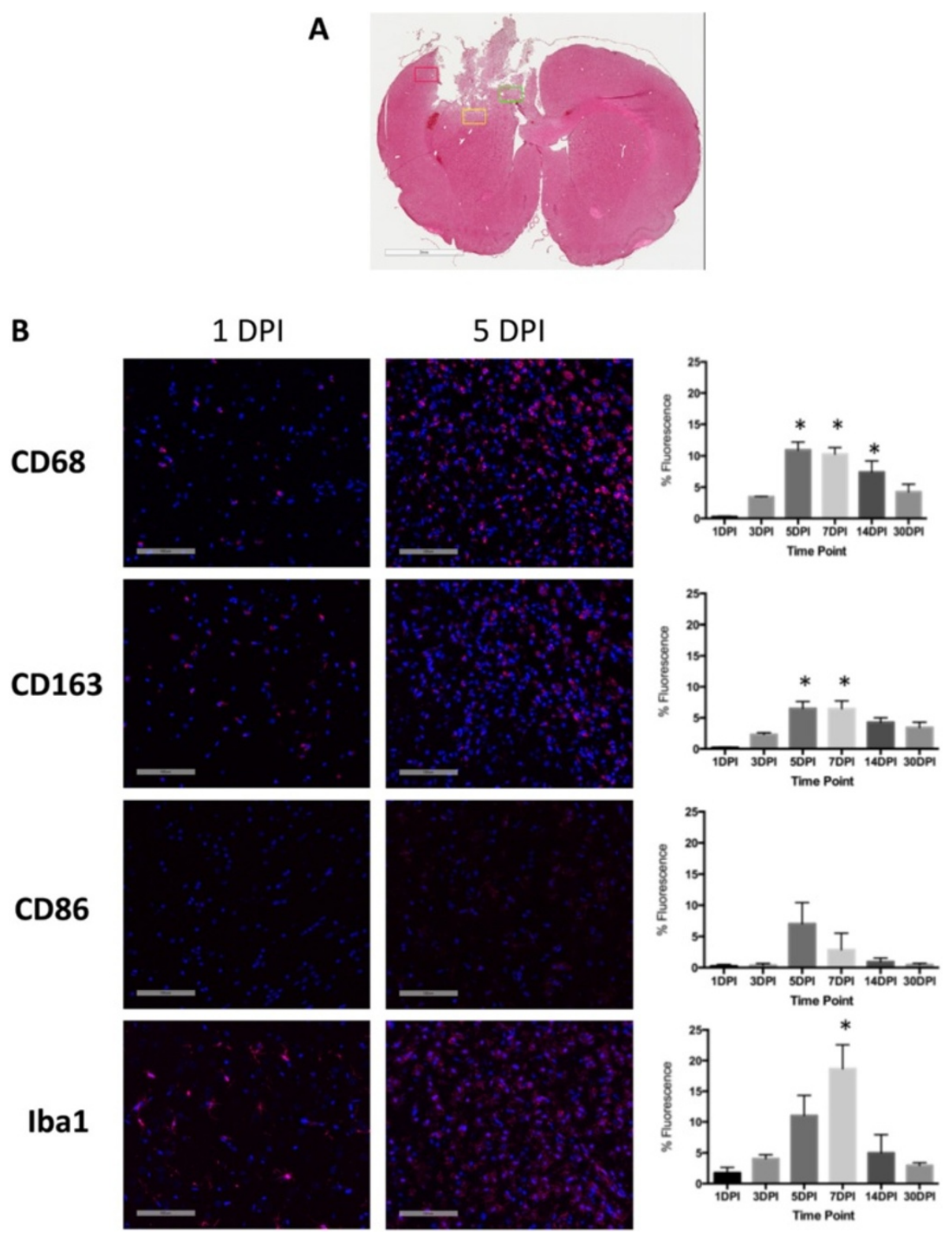

Figure 4 Immunohistochemistry of macrophagic and microglial markers in brain tissue after traumatic brain injury over time. Maximum immunofluorescence detection of markers of macrophagic and microglial polarization occurred by 5 days postinjury (DPI). (A) Representative anatomical localization of the regions of interest (ROls; rectangles) quantified for the immunofluorescence of each marker. These ROls were examined for the same anatomical slices from each animal. Scale bar $=3 \mathrm{~mm}$. (B) Quantification of immunofluorescence in ROls and representative immunofluorescent images at 1 DPI and 5 DPI. For a given time point, the column represents the average of all three ROls for each slice, as shown in (A). Iba1, lonized calcium-binding adapter molecule 1. Error bars indicate standard errors of the mean. ${ }^{*} P<0.05$ for time point relative to $1 \mathrm{DPI}$. Scale bars $=100 \mu \mathrm{m}$.

Researchers in two studies who used a mouse TBI model reported a mixed $\mathrm{M} 1$ and $\mathrm{M} 2$ response after $\mathrm{CCI}$ in male mice $[34,35]$, with the M2 response peaking at 5 days and then rapidly decreasing by 1 week postinjury, with a return to baseline by 2 weeks. Zhang et al. [36] reported their use of the open-skull weight-drop model of TBI in Lewis rats (sex was not specified). They found parenchymal accumulation of M2 marker CD163 macrophages in the lesion between days 2 and 4 postinjury [36]; however, they obtained no data at time points beyond 96 hours. In our present study, CD163 expression peaked at 5 days. The slight temporal shifts between the 


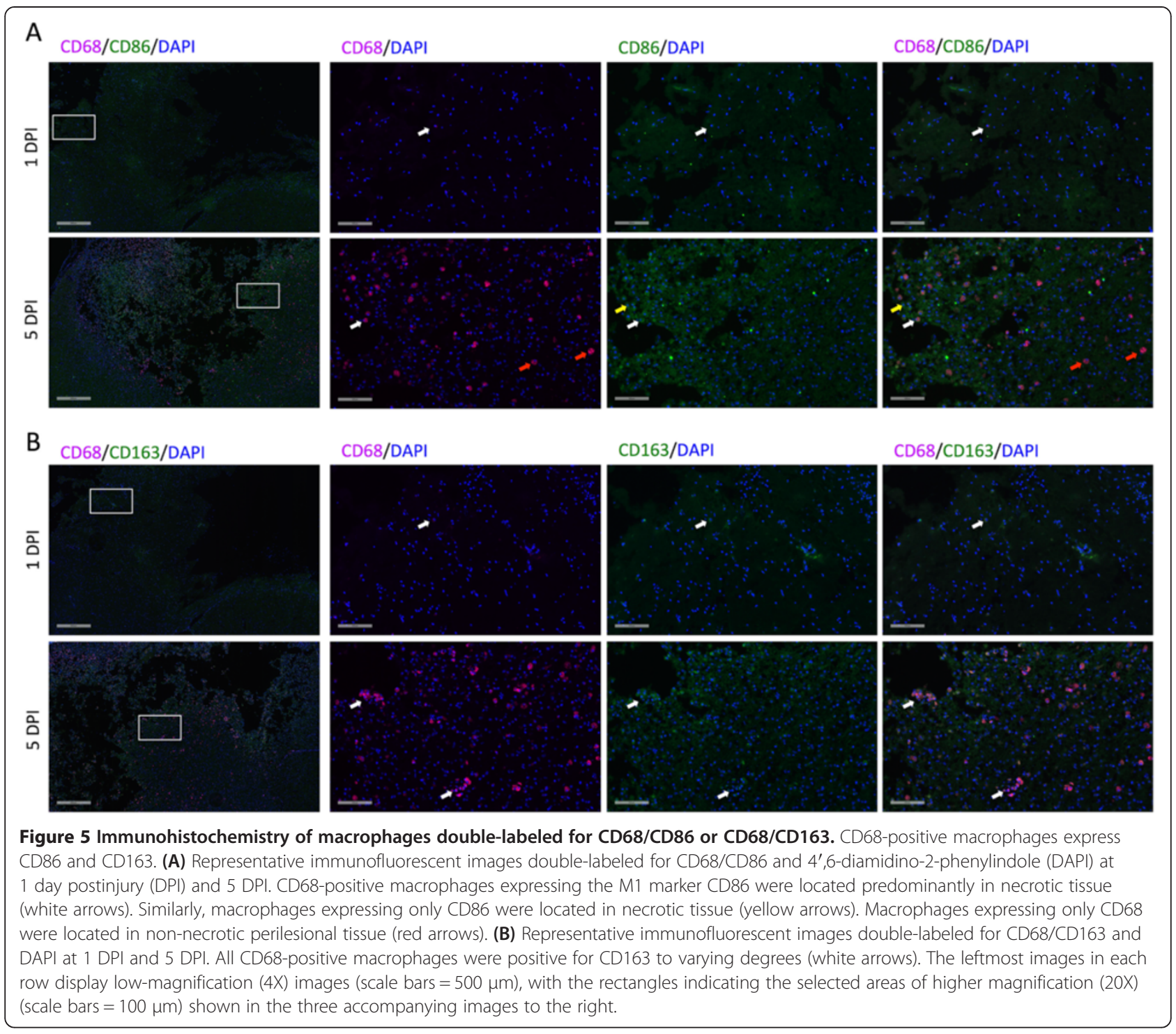

diffuse weight-drop study [36] and our present study may be due in part to the different injury mechanisms and/or strain differences.

Researchers in several studies of mice and rats have investigated additional aspects of the neuroinflammatory response after experimental TBI. Lagraoui et al. [32] reported that CCI in male C56BL/6 mice induced a similar level of injury severity over a similar location (the motor cortex) compared to those in our study, and they examined the kinetics of the subsequent inflammatory response. Similarly to our present findings in rats, they found that RNA expression of CXCL1 and the proinflammatory cytokine IL- $1 \beta$ increased in expression early in the C56BL/6 mouse [32]. Peak expression of M1 marker CD86 in their study appeared closer to 7 days postinjury at the injury site compared to the 5 DPI peak observed in our present study. Their analysis of cytokine and chemokine expression by multiplex analysis postCCI in the C56BL/6 mouse model showed similar upregulation of CXCL1 at 1 DPI. In contrast to the results of our present study, CCI in the mouse in their study resulted in significantly increased expression of IL-6, IL$1 \beta$, IL-10, IL-12p70 and IFN $\gamma$ protein in their time course [32].

Kumar et al. [15] reported that, at 24 hours after CCI, substantial upregulation of RNA relative to sham controls for CD86, IL-1 $\beta, \mathrm{TNF} \alpha$, Arg1 and inducible Nos2 in male mice ages 24 months and 3 months. Similar upregulation of TNF $\alpha$ and IL- $1 \beta$ RNA expression was found in the present study at 24 hours after CCI. Similar to young male mice in a previous study [15], the young female rats in our present study had little change in CD86 at 24 hours after CCI relative to controls. In contrast, by 5 DPI in female rats, we observed substantial 


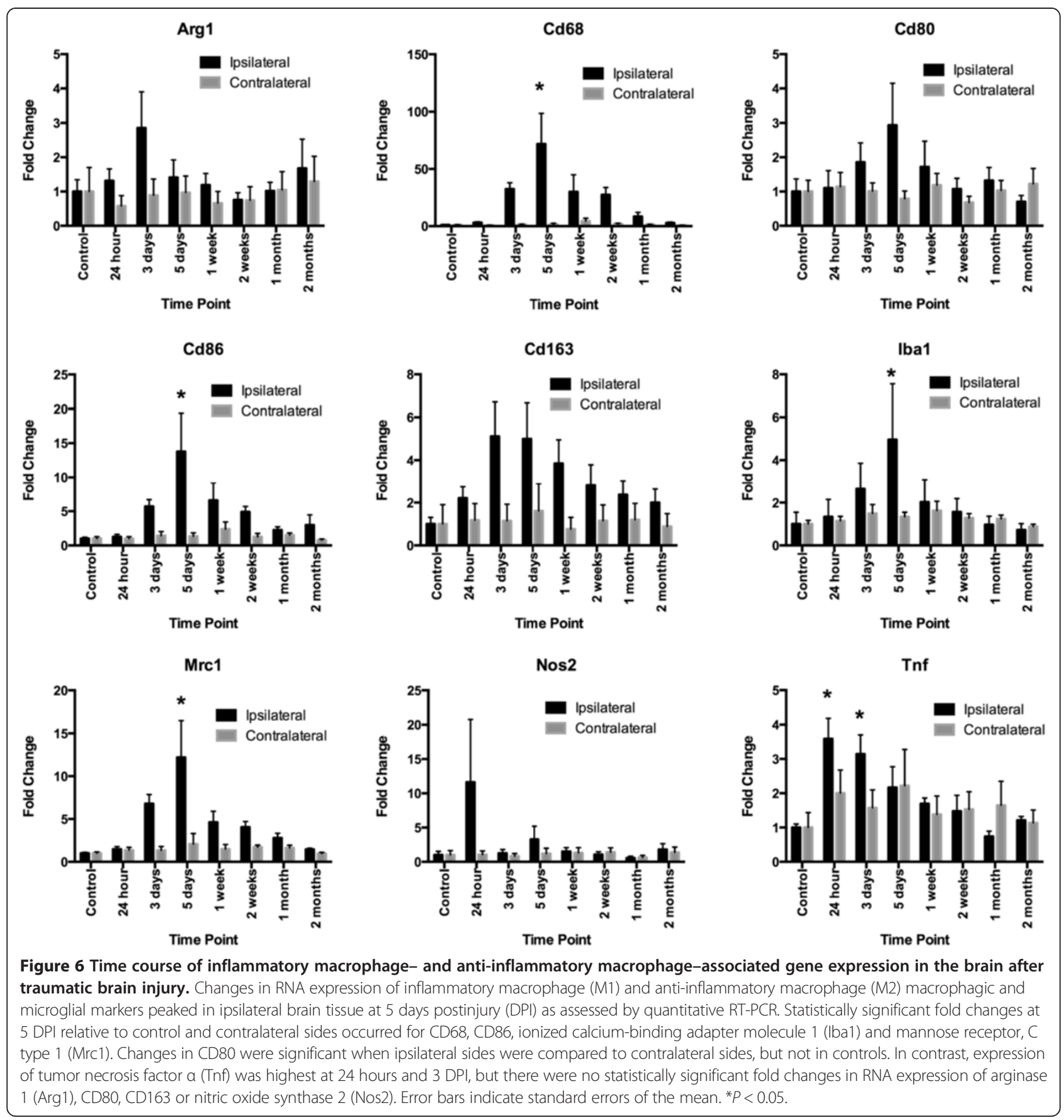

fold changes in RNA expression by 5 DPI for CD68, CD86, Iba1 and Mrc1 (Figure 6). Examination of the RNA expression data from young male mice in a previous study [15] demonstrated few differences between young injured animals and controls at 24 hours postinjury. It is unknown whether older female rats would show upregulation of both pro- and anti-inflammatory markers at 24 hours postinjury, as was seen previously in older male mice [15].
At 24 hours post-CCI, previous authors reported that there was upregulation of $\mathrm{KC} / \mathrm{GRO}$ in TBI relative to control male Sprague-Dawley rats [33]. In the present study, macrophage infiltration was not observed prior to 24 hours postinjury; therefore, we did not obtain data at earlier time points. The global decreases in protein expression in ipsilateral brain tissues relative to the contralateral side and to the control female rats observed were different from those reported in previous studies, which 


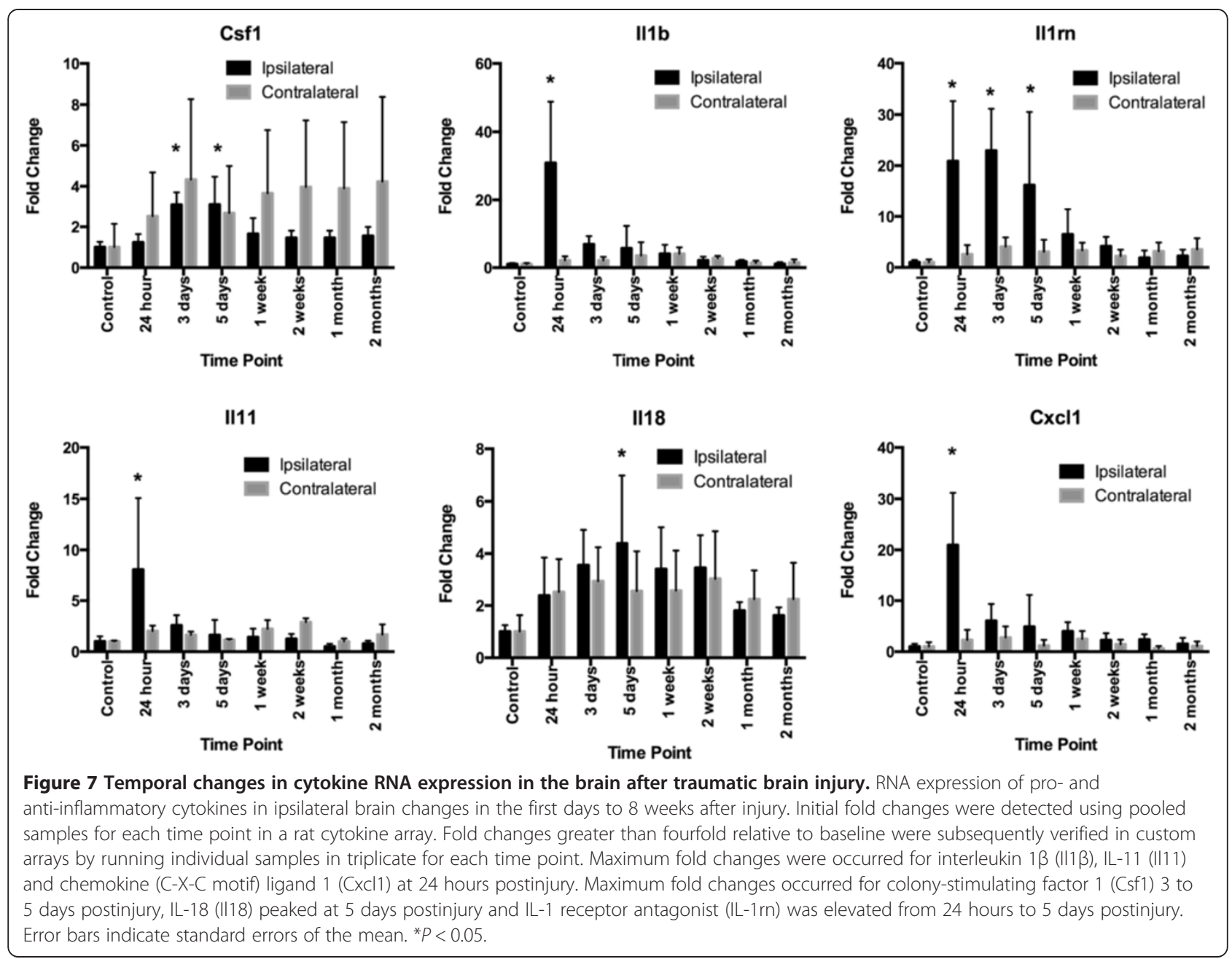

may reflect technical, strain or sex differences in response to TBI [33]. For example, Holmin et al. [37] used an open-skull (posterior) craniotomy weight-drop model in female Sprague-Dawley rats to investigate an inflammatory time course after injury. By in situ hybridization and immunohistochemistry, no expression of IL-1 $\beta$, TNF $\alpha$, IL- 6 or IFN $\gamma$ was detected within the first 2 days after injury. By days 4 to 6 postinjury, strong IL-1 $\beta$, TNF $\alpha$ and IL-6 mRNA was detected by in situ hybridization [37], indicating a delayed response. In contrast, after a penetrating ballistic injury (PBI) in male Sprague-Dawley rats, upregulation of IL-1 $\beta$, IL-6, IFN $\gamma$ and TNF $\alpha$ by protein multiplex analysis was observed from 4 hours to 3 DPI in a previous study [38]. In the PBI model, neutrophil infiltration occurs at 24 hours postinjury, with a maximum microglial response by 72 hours [39], in contrast to our findings in our present study using the CCI model, in which the microglial response appeared to peak at 5 to 7 days. The differences in time course of cytokine expression in these different pathogenic TBI models [37-39] suggest considerable heterogeneity of inflammatory responses after TBI.

Multiple factors complicate the results obtained from the use of these different methods in our present study. Flow cytometry was performed on samples after enrichment using OptiPrep gradient medium to select for inflammatory cells, whereas neurons, oligodendrocytes and astrocytes were in nonanalyzed fractions of the gradient. In this cell population highly enriched for inflammatory cells, flow cytometry revealed that more than $50 \%$ of cells stained positive for CD68 and/or CD40 and less than 20\% expressed CD163 at peak (Figure 3). In contrast, the brain slices used for IHC contained a heterogeneous cell population, diluting the concentration of immunological cells. Our comparisons were also complicated by the use of different M1 marker antibodies in the flow cytometry studies (CD40) and in IHC (CD86). A CD86 antibody for use in the rat was not available prior to the completion of the flow cytometry arm of this study. Initially, the M1 marker CD40 was also tested 


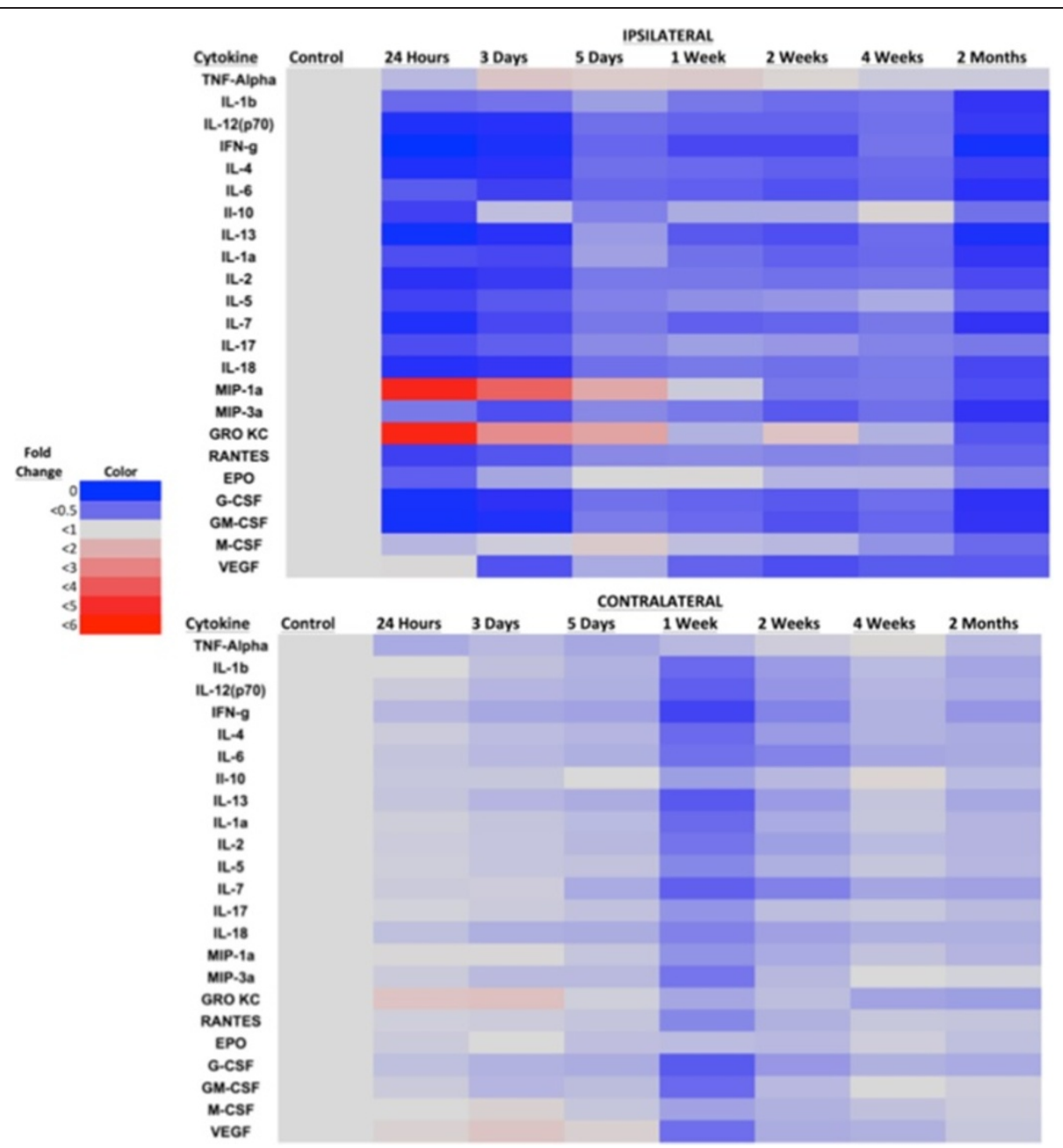

Figure $\mathbf{8}$ Changes in cytokine and chemokine protein expression after traumatic brain injury. The fold change protein expression of cytokines and growth factors assessed by multiplex assay decreased relative to expression in naïve controls in the combined lesion/perilesion area after injury, as summarized in the accompanying heat map. Chemokines macrophage inflammatory protein 1a (MIP-1a) and keratinocyte chemoattractant/growth-related oncogene (GRO KC) were the only proteins observed to have increased over the time course, peaking at 24 hours after injury, then decreasing. EPO, Erythropoietin; G-CSF, Granulocyte colony-stimulating factor; GM-CSF, Granulocyte-macrophage colony-stimulating factor; IFN-g, Interferon Y; IL, Interleukin; M-CSF, Macrophage colony-stimulating factor; RANTES, Regulated on activation, normal T cell expressed and secreted; TNF, Tumor necrosis factor; VEGF, Vascular endothelial growth factor. ${ }^{*} P<0.05$.

by IHC. Over the time course examined, however, the antibody demonstrated a high degree of background staining in nonmicroglial, nonmacrophagic cells in brain tissue (data not shown) in contrast to the enriched fraction of cells analyzed by flow cytometry, where minimal background staining was observed in the enriched cell population with antibody isotype controls (Figure 3B). The M1 marker CD86 subsequently used for IHC was more specific for macrophages and microglia in tissue sections.

For the flow cytometry arm of these investigations, the choice of species limited options for antibodies that could be used to further differentiate and gate cell populations. In mice, the antibody Ly6G can be used to exclude the neutrophil population and a CD45 antibody can distinguish macrophages (high CD45 expression) from microglia (low CD45 expression) [34,40]. Although a CD45 antibody is now available in rats, Ly6G is not. In addition, there are many more mouse antibodies available to distinguish M1 and M2 phenotypes than are currently available for rats (Table 1). A more refined cell sorting and gating strategy similar to that used by Bedi et al. [40] to separate macrophages $(\mathrm{CD} 11 \mathrm{~b}+$, rat analogue to Ly6G $\mathrm{CD} 45+$ (high expression)) from microglia $(\mathrm{CD} 11 \mathrm{~b}+$, rat analogue to Ly6G-CD45+ (low expression)) would increase sensitivity for future flow cytometry studies to investigate $\mathrm{M} 1$ and $\mathrm{M} 2$ responses.

There is currently no antibody available in the rat that will exclusively identify microglia from macrophages. Although enrichment strategies can be attempted for flow 
Table 1 Antibody studies of M1 versus M2 phenotypes in neurotrauma ${ }^{a}$

\begin{tabular}{|c|c|c|c|c|c|c|c|}
\hline Study & $\begin{array}{l}\text { Injury } \\
\text { model }\end{array}$ & Species & Sex & Methods & $\begin{array}{l}\text { Cell type } \\
\text { antibodies }\end{array}$ & $\begin{array}{c}\mathrm{M} 1 \\
\text { markers }\end{array}$ & $\begin{array}{c}\text { M2 } \\
\text { markers }\end{array}$ \\
\hline Bedi et al. [40] & $\mathrm{CCl}$ & Mouse & Male & FC & CD11b, CD45 & CD16/CD32 & CD206 \\
\hline Hsieh et al. [34] & $\mathrm{CCl}$ & Mouse & Male & $\begin{array}{l}\text { FC, qRT-PCR on } \\
\text { FC-sorted cells }\end{array}$ & $\begin{array}{l}\text { CD11b, CD45, } \\
\text { F4/F80, Ly6G }\end{array}$ & CD86, MHC II, YFP-IL-12p40 & YFP-Arg1 \\
\hline Kigerl et al. [9] & $\mathrm{SCl}$ & Mouse & Not specified & $\mathrm{IHC}$, qRT-PCR & None & $\begin{array}{c}\text { CD16/CD32, CD86, } \\
\text { iNOS, MHC II }\end{array}$ & Arg1, CD206 \\
\hline Kumar et al. [15] & $\mathrm{CCl}$ & Mouse & Male & IHC, qRT-PCR & CD11b, CD68, Iba1 & $\mathrm{MHC} \|$ & $Y m 1$ \\
\hline Lagraoui et al. [32] & $\mathrm{CCl}$ & Mouse & Male & qRT-PCR, multiplex & None & None & None \\
\hline Present study & $\mathrm{CCl}$ & Rat & Female & FC, IHC, qRT-PCR, multiplex & CD68, Iba1 & CD40, CD86 & CD163 \\
\hline Wang et al. [35] & $\mathrm{CCl}$ & Mouse & Male & IHC, qRT-PCR & Iba1 & CD16/CD32 & CD206 \\
\hline Zhang et al. [36] & $\mathrm{CCl}$ & Rat & Not specified & $\mathrm{IHC}$ & CD68 & None & CD163 \\
\hline
\end{tabular}

${ }^{a}$ Arg1, Arginine 1; CCl, Controlled cortical impact; FC = Flow cytometry; Iba1, lonized calcium-binding adapter molecule 1; IHC, Immunohistochemistry; IL, Interleukin; iNOS, Inducible nitric oxide synthase; MHC II, Major histocompatibility complex class II; $q R T-P C R$, Quantitative RT-PCR; SCI = Spinal cord injury, YFP $=$ Yellow fluorescent protein.

cytometry studies, technical limitations currently make it difficult to distinguish between macrophages and microglia in a mixed population of cells, such as the brain tissue slices used for IHC or the brain tissue cores used for qRT-PCR in the present study. If an antibody that can reliably distinguish between macrophages and microglia is found, additional details related to M1 and M2 responses specifically in these cells in both the lesion and perilesion areas might be possible.

Factors underlying the observed differences in cytokine expression between the present study and others published in the literature include species (rat versus mouse), strain and sex differences as well as different methodologies. Methodological differences in the animal models (that is, depth of injury, CCI location, amount of hemorrhage at site of CCI), depending on species $[24,32]$ and techniques (that is, tissue isolation, fresh versus flash frozen tissue for analysis) [33] used in tissue isolation and processing, may contribute to and explain differences between previously reported results and the results in the present study.

Genetic heterogeneity can also contribute to different outcomes after TBI [41,42] and can manifest as differences in immunological response. Nimer et al. [43] investigated genetic differences between two strains of inbred rats (Piebald Virol Glaxo (PVG) and Dark Agouti (DA)) that share similar major histocompatibility haplotypes but manifested differences in neuronal survival after TBI. Nimer et al. observed higher degrees of microglial and macrophagic activation, as well as greater activation of other immune cells, in the DA strain compared to the PVG strain in the CCI region. Genetic differences between strains are likely to be present to a greater degree in outbred strains, such as Wistar or Sprague-Dawley rats, commonly used in CCI studies.

In addition to genetic variability between strains and species, comparisons of results between studies can be confounded further by sex differences in the animals used. The response to neuroinflammation is regulated by estrogen levels via estrogen receptors [44]. Estrogen has been shown to have inhibitory effects on proinflammatory cytokines after diffuse TBI in rats [45]. Although the preinjury estrous stage has no correlation with outcome after experimental TBI in rats [46], female rats have better shortterm behavioral outcomes after injury than males, suggesting that exposure to endogenous circulating sex steroids confers some neuroprotection against TBI. Whether TBI itself changes postinjury estrogen and progesterone levels in experimental animals is unknown; however, in clinical populations, both acute and chronic hypogonadotropic hypogonadism are known phenomena [47].

A differential neuroinflammatory response secondary to the short- and long-term effects of sex steroids may explain differences noted in studies of TBI in male versus female rodents. After experimental TBI, male rats have more brain edema [48] and brain injury [49] than cycling female rats, whereas ovariectomized female rats have levels of edema and injury similar to those of males. In gonadally intact female rats, estrogen and progesterone levels vary during the course of the 4- to 5day estrous cycle [50,51]. After experimental cortical injury, pseudopregnant female rats, which have prolonged elevated progesterone levels, have less edema than normally cycling females [52]. Progesterone supplementation reduces edema and injury after experimental TBI in both female and male rats [48,53-57]. These preclinical studies suggest that exposure to sex steroid hormones, especially progesterone, can influence the response to TBI in experimental models.

\section{Conclusions}

The present study demonstrates the complexity of the macrophagic and microglial responses after CCI in female Wistar rats. The brain microenvironment has elements of 
both pro- and anti-inflammatory responses within the first week after traumatic injury, rather than one that is clearly skewed more toward an M1 or M2 phenotype. A spectrum of macrophagic and microglial phenotypes exists after CCI, likely reflecting a complex inflammatory response in which the cells may adjust their functions based upon the posttraumatic milieu. This mixed pattern has relevance to translation of results to clinical populations. Future studies are needed to address the contributions of strain, species, sex and age differences to the neuroinflammatory response after TBI.

\section{Additional file}

Additional file 1: Figure S1. Protein concentrations of cytokines and chemokines after traumatic brain injury. After traumatic brain injury (TBI), the protein concentrations of most cytokines, chemokines and growth factors decreased on the side ipsilateral to TBI as assessed by Bio-Plex multiplex system panel (Bio-Rad Laboratories, Hercules, CA, USA). EPO, Erythropoietin; G-CSF, Granulocyte colony-stimulating factor; GM-CSF, Granulocyte-macrophage colony-stimulating factor; GRO KC, Growthrelated oncogene (also known as chemokine (C-X-C motif) ligand 1); IFN, Interferon; IL, Interleukin; M-CSF, Macrophage colony-stimulating factor; MIP, Macrophage inflammatory protein; RANTES, Regulated on activation, normal T cell expressed and secreted; TNF, Tumor necrosis factor; VEGF, Vascular endothelial growth factor. Error bars indicate standard errors of the mean. ${ }^{*} P<0.05$ for the indicated ipsilateral side time point relative to the control value. ${ }^{* *} P<0.05$ for the ipsilateral side versus the contralateral side.

\section{Abbreviations}

ANOVA: Analysis of variance; Arg1: Arginase 1; CCl: Controlled cortical impact; CXCL1: Chemokine (C-X-C motif) ligand 1 (also known as Growthrelated oncogene); DA: Dark agouti rat strain; DAPI: 4,6-diamidino-2phenylindole; DPI: Days postinjury; ELISA: Enzyme-linked immunosorbent assay; H \& E: Hematoxylin and eosin; Iba1: Ionized calcium-binding adapter molecule 1; IHC: Immunohistochemistry; IL: Interleukin; KC/GRO: Keratinocyte chemoattractant/growth-related oncogene (also known as chemokine (C-X-C motif) ligand 1); MIP-1a: Macrophage inflammatory protein 1a; Mrc1: Mannose receptor, C type 1; MRI: Magnetic resonance imaging; Nos2: Nitric oxide synthase 2; PBS: Phosphate-buffered saline; PVG: Piebald Virol Glaxo rat strain; qRT-PCR: Quantitative real-time polymerase chain reaction; ROI: Region of interest; SCl: Spinal cord injury; TBI: Traumatic brain injury; TNFa: Tumor necrosis factor a.

\section{Competing interests}

The authors declare that they have no competing interests.

\section{Authors' contributions}

LCT participated in the design and coordination of the study, performed animal experimental procedures, conducted flow cytometry and molecular analyses and drafted the manuscript. JL conducted immunohistochemical analyses. $\downarrow$ performed animal experimental procedures and conducted ex vivo MRI and immunohistochemical analyses. DDD performed animal experimental procedures, designed the immunohistochemistry protocols and assisted in data analysis and interpretation. MDB designed the ex vivo MRI protocols. JAF conceived of the study, participated in its design and helped draft the manuscript. All authors read and approved the final manuscript.

\section{Acknowledgements}

These studies were supported by the US Department of Defense Center for Neuroscience and Regenerative Medicine under Henry M. Jackson Foundation for the Advancement of Military Medicine award 300604-13.0160855 and by the National Institutes of Health Intramural Research Program. We would also like to thank Aneeka Chaudhry, Tiziana Coppola and Eric Gold for their invaluable technical assistance with these studies.

\section{Author details}

${ }^{1}$ Center for Neuroscience and Regenerative Medicine, Uniformed Services University of the Health Sciences, 4301 Jones Bridge Road, Bethesda, MD 20814, USA. ${ }^{2}$ Frank Laboratory, National Institutes of Health, Building 10, Room B1N256, MSC 1074, 10 Center Drive, Bethesda, MD 20814, USA. ${ }^{3}$ National Institute of Biomedical Imaging and Bioengineering, National Institutes of Health, 10 Center Drive, Bethesda, MD 20814, USA. ${ }^{4}$ Department of Environmental Science, Baylor University, One Bear Place \#97266, Waco, TX 76798, USA. ${ }^{5}$ Department of Neurosurgery, Medical College of Wisconsin, 8701 Watertown Plank Road, Milwaukee, WI 53226, USA.

Received: 3 February 2014 Accepted: 6 April 2014

Published: 24 April 2014

\section{References}

1. Faul M, Xu L, Wald MM, Coronado VG: Traumatic Brain Injury in the United States: Emergency Department Visits, Hospitalizations and Deaths 2002-2006. Atlanta, GA: US Department of Health and Human Services, Centers for Disease Control and Prevention, National Center for Injury Prevention and Control; 2010. Available at http://www.cdc.gov/traumaticbraininjury/pdf/ blue_book.pdf; (accessed 15 April 2014).

2. Bell RS, Vo AH, Neal CJ, Tigno J, Roberts R, Mossop C, Dunne JR, Armonda RA: Military traumatic brain and spinal column injury: a 5-year study of the impact blast and other military grade weaponry on the central nervous system. J Trauma 2009, 66(4 Suppl):S104-S111.

3. Roberts I, Schierhout G, Alderson P: Absence of evidence for the effectiveness of five interventions routinely used in the intensive care management of severe head injury: a systematic review. J Neurol Neurosurg Psychiatry 1998, 65:729-733.

4. Kumar A, Loane DJ: Neuroinflammation after traumatic brain injury: opportunities for therapeutic intervention. Brain Behav Immun 2012, 26:1191-1201.

5. Komohara Y, Ohnishi K, Kuratsu J, Takeya M: Possible involvement of the M2 anti-inflammatory macrophage phenotype in growth of human gliomas. J Pathol 2008, 216:15-24.

6. Mantovani A, Sica A, Sozzani S, Allavena P, Vecchi A, Locati M: The chemokine system in diverse forms of macrophage activation and polarization. Trends Immunol 2004, 25:677-686.

7. Mantovani A, Sozzani S, Locati M, Allavena P, Sica A: Macrophage polarization: tumor-associated macrophages as a paradigm for polarized M2 mononuclear phagocytes. Trends Immunol 2002, 23:549-555.

8. Ydens E, Cauwels A, Asselbergh B, Goethals S, Peeraer L, Lornet G, AlmeidaSouza L, Van Ginderachter JA, Timmerman V, Janssens $S$ : Acute injury in the peripheral nervous system triggers an alternative macrophage response. J Neuroinflammation 2012, 9:176.

9. Kigerl KA, Gensel JC, Ankeny DP, Alexander JK, Donnelly DJ, Popovich PG: Identification of two distinct macrophage subsets with divergent effects causing either neurotoxicity or regeneration in the injured mouse spinal cord. J Neurosci 2009, 29:13435-13444.

10. Sica A, Schioppa T, Mantovani A, Allavena P: Tumour-associated macrophages are a distinct M2 polarised population promoting tumour progression: potential targets of anti-cancer therapy. Eur J Cancer 2006, 42:717-727.

11. Popovich $P G$, Longbrake EE: Can the immune system be harnessed to repair the CNS? Nat Rev Neurosci 2008, 9:481-493.

12. Stout RD, Suttles J: Functional plasticity of macrophages: reversible adaptation to changing microenvironments. J Leukoc Biol 2004, 76:509-513.

13. Stout RD, Jiang C, Matta B, Tietzel I, Watkins SK, Suttles J: Macrophages sequentially change their functional phenotype in response to changes in microenvironmental influences. J Immunol 2005, 175:342-349.

14. Colton CA: Heterogeneity of microglial activation in the innate immune response in the brain. J Neuroimmune Pharmacol 2009, 4:399-418.

15. Kumar A, Stoica BA, Sabirzhanov B, Burns MP, Faden Al, Loane DJ: Traumatic brain injury in aged animals increases lesion size and chronically alters microglial/macrophage classical and alternative activation states. Neurobiol Aging 2013, 34:1397-1411.

16. Ding AH, Nathan CF, Stuehr DJ: Release of reactive nitrogen intermediates and reactive oxygen intermediates from mouse peritoneal macrophages: comparison of activating cytokines and evidence for independent production. J Immunol 1988, 141:2407-2412. 
17. Stein M, Keshav S, Harris N, Gordon S: Interleukin 4 potently enhances murine macrophage mannose receptor activity: a marker of alternative immunologic macrophage activation. J Exp Med 1992, 176:287-292.

18. Bonecchi R, Facchetti F, Dusi S, Luini W, Lissandrini D, Simmelink M, Locati M, Bernasconi S, Allavena P, Brandt E, Rossi F, Mantovani A, Sozzani S: Induction of functional IL-8 receptors by IL-4 and IL-13 in human monocytes. J Immunol 2000, 164:3862-3869.

19. Donnelly DJ, Popovich PG: Inflammation and its role in neuroprotection, axonal regeneration and functional recovery after spinal cord injury. Exp Neurol 2008, 209:378-388.

20. Dixon CE, Clifton GL, Lighthall JW, Yaghmai AA, Hayes RL: A controlled cortical impact model of traumatic brain injury in the rat. $J$ Neurosci Methods 1991, 39:253-262

21. Kochanek PM, Marion DW, Zhang W, Schiding JK, White M, Palmer AM Clark RS, O'Malley ME, Styren SD, Ho C, DeKosky ST: Severe controlled cortical impact in rats: assessment of cerebral edema, blood flow, and contusion volume. J Neurotrauma 1995, 12:1015-1025

22. Clark RS, Kochanek PM, Dixon CE, Chen M, Marion DW, Heineman S, DeKosky ST, Graham SH: Early neuropathologic effects of mild or moderate hypoxemia after controlled cortical impact injury in rats. J Neurotrauma 1997, 14:179-189.

23. Brody DL, Mac Donald C, Kessens CC, Yuede C, Parsadanian M, Spinner M, Kim E, Schwetye KE, Holtzman DM, Bayly PV: Electromagnetic controlled cortical impact device for precise, graded experimental traumatic brain injury. J Neurotrauma 2007, 24:657-673.

24. Turtzo LC, Budde MD, Gold EM, Lewis BK, Janes L, Yarnell A, Grunberg NE, Watson W. Frank JA: The evolution of traumatic brain injury in a rat focal contusion model. NMR Biomed 2013, 26:468-479.

25. Beck KD, Nguyen HX, Galvan MD, Salazar DL, Woodruff TM, Anderson AJ: Quantitative analysis of cellular inflammation after traumatic spinal cord injury: evidence for a multiphasic inflammatory response in the acute to chronic environment. Brain 2010, 133:433-447.

26. Cole JT, Yarnell A, Kean WS, Gold E, Lewis B, Ren M, McMullen DC, Jacobowitz DM, Pollard HB, O'Neill JT, Grunberg NE, Dalgard CL, Frank JA, Watson WD: Craniotomy: true sham for traumatic brain injury, or a sham of a sham? J Neurotrauma 2011, 28:359-369.

27. Jacobowitz DM, Cole JT, McDaniel DP, Pollard HB, Watson WD: Microglia activation along the corticospinal tract following traumatic brain injury in the rat: a neuroanatomical study. Brain Res 2012, 1465:80-89.

28. Chung IY, Benveniste EN: Tumor necrosis factor-alpha production by astrocytes: induction by lipopolysaccharide, IFN- $\gamma$, and IL-1 $\beta$. J Immunol 1990, 144:2999-3007.

29. Liu T, Clark RK, McDonnell PC, Young PR, White RF, Barone FC, Feuerstein GZ: Tumor necrosis factor-a expression in ischemic neurons. Stroke 1994 25:1481-1488

30. McKimmie CS, Graham GJ: Astrocytes modulate the chemokine network in a pathogen-specific manner. Biochem Biophys Res Commun 2010, 394:1006-1011.

31. Liu $L$, Chan C: IPAF inflammasome is involved in interleukin-1 $\beta$ production from astrocytes, induced by palmitate: implications for Alzheimer's disease. Neurobiol Aging 2014, 35:309-321.

32. Lagraoui M, Latoche JR, Cartwright NG, Sukumar G, Dalgard CL, Schaefer BC: Controlled cortical impact and craniotomy induce strikingly similar profiles of inflammatory gene expression, but with distinct kinetics. Front Neurol 2012, 3:155.

33. Dalgard CL, Cole JT, Kean WS, Lucky JJ, Sukumar G, McMullen DC, Pollard $H B$, Watson WD: The cytokine temporal profile in rat cortex after controlled cortical impact. Front Mol Neurosci 2012, 5:6.

34. Hsieh CL, Kim CC, Ryba BE, Niemi EC, Bando JK, Locksley RM, Liu J, Nakamura MC, Seaman WE: Traumatic brain injury induces macrophage subsets in the brain. Eur J Immunol 2013, 43:2010-2022.

35. Wang G, Zhang J, Hu X, Zhang L, Mao L, Jiang X, Liou AK, Leak RK, Gao Y, Chen J: Microglia/macrophage polarization dynamics in white matter after traumatic brain injury. J Cereb Blood Flow Metab 2013, 33:1864-1874.

36. Zhang Z, Zhang ZY, Wu Y, Schluesener HJ: Lesional accumulation of $\mathrm{CD}_{163^{+}}$macrophages/microglia in rat traumatic brain injury. Brain Res 2012, 1461:102-110.

37. Holmin S, Schalling M, Höjeberg B, Nordqvist AC, Skeftruna AK, Mathiesen T: Delayed cytokine expression in rat brain following experimental contusion. J Neurosurg 1997, 86:493-504.
38. Wei HH, Lu XC, Shear DA, Waghray A, Yao C, Tortella FC, Dave JR: NNZ-2566 treatment inhibits neuroinflammation and pro-inflammatory cytokine expression induced by experimental penetrating ballistic-like brain injury in rats. J Neuroinflammation 2009, 6:19.

39. Williams AJ, Wei HH, Dave JR, Tortella FC: Acute and delayed neuroinflammatory response following experimental penetrating ballistic brain injury in the rat. J Neuroinflammation 2007, 4:17.

40. Bedi SS, Smith P, Hetz RA, Xue H, Cox CS: Immunomagnetic enrichment and flow cytometric characterization of mouse microglia. J Neurosci Methods 2013, 219:176-182.

41. Jordan BD: Genetic influences on outcome following traumatic brain injury. Neurochem Res 2007, 32:905-915.

42. Cederberg D, Siesjö P: What has inflammation to do with traumatic brain injury? Childs Nerv Syst 2010, 26:221-226.

43. Al Nimer F, Lindblom R, Ström M, Guerreiro-Cacais AO, Parsa R, Aeinehband S, Mathiesen T, Lidman O, Piehl F: Strain influences on inflammatory pathway activation, cell infiltration and complement cascade after traumatic brain injury in the rat. Brain Behav Immun 2013, 27:109-122.

44. Brown CM, Mulcahey TA, Filipek NC, Wise PM: Production of proinflammatory cytokines and chemokines during neuroinflammation: novel roles for estrogen receptors $\alpha$ and $\beta$. Endocrinology 2010, 151:4916-4925.

45. Khaksari M, Keshavarzi Z, Gholamhoseinian A, Bibak B: The effect of female sexual hormones on the intestinal and serum cytokine response after traumatic brain injury: different roles for estrogen receptor subtypes. Can J Physiol Pharmacol 2013, 91:700-707.

46. Wagner AK, Willard LA, Kline AE, Wenger MK, Bolinger BD, Ren D, Zafonte $\mathrm{RD}$, Dixon CE: Evaluation of estrous cycle stage and gender on behavioral outcome after experimental traumatic brain injury. Brain Res 2004, 998:113-121.

47. Wagner AK, Brett CA, McCullough EH, Niyonkuru C, Loucks TL, Dixon CE, Ricker J, Arenth P, Berga SL: Persistent hypogonadism influences estradiol synthesis, cognition and outcome in males after severe TBI. Brain Inj 2012, 26:1226-1242.

48. Roof RL, Duvdevani R, Stein DG: Gender influences outcome of brain injury: progesterone plays a protective role. Brain Res 1993, 607:333-336.

49. Bramlett HM, Dietrich WD: Neuropathological protection after traumatic brain injury in intact female rats versus males or ovariectomized females. J Neurotrauma 2001, 18:891-900.

50. Butcher RL, Collins WE, Fugo NW: Plasma concentration of LH, FSH, prolactin, progesterone and estradiol-17 $\beta$ throughout the 4-day estrous cycle of the rat. Endocrinology 1974, 94:1704-1708.

51. de Greef WJ, Zeilmaker GH: Blood progesterone levels in pseudopregnant rats: effects of partial removal of luteal tissue. Endocrinology 1974, 95:565-571.

52. Attella MJ, Nattinville A, Stein DG: Hormonal state affects recovery from frontal cortex lesions in adult female rats. Behav Neural Biol 1987, 48:352-367.

53. Roof RL, Duvdevani R, Braswell L, Stein DG: Progesterone facilitates cognitive recovery and reduces secondary neuronal loss caused by cortical contusion injury in male rats. Exp Neurol 1994, 129:64-69.

54. Roof RL, Duvdevani R, Heyburn JW, Stein DG: Progesterone rapidly decreases brain edema: treatment delayed up to 24 hours is still effective. Exp Neurol 1996, 138:246-251.

55. Robertson CL, Puskar A, Hoffman GE, Murphy AZ, Saraswati M, Fiskum G: Physiologic progesterone reduces mitochondrial dysfunction and hippocampal cell loss after traumatic brain injury in female rats. Exp Neurol 2006, 197:235-243.

56. Cutler SM, Cekic M, Miller DM, Wali B, VanLandingham JW, Stein DG: Progesterone improves acute recovery after traumatic brain injury in the aged rat. J Neurotrauma 2007, 24:1475-1486.

57. Shahrokhi N, Khaksari M, Soltani Z, Mahmoodi M, Nakhaee N: Effect of sex steroid hormones on brain edema, intracranial pressure, and neurologic outcomes after traumatic brain injury. Can J Physiol Pharmacol 2010, 88:414-421.

doi:10.1186/1742-2094-11-82

Cite this article as: Turtzo et al.: Macrophagic and microglial responses

after focal traumatic brain injury in the female rat. Journal of Neuroinflammation 2014 11:82. 